\title{
Critical Review Of Quark Gluon Plasma Signals *
}

\author{
D. Zschiesche ${ }^{\mathrm{a}}$, L. Gerland ${ }^{\mathrm{a}}, \mathrm{S}$. Schramm ${ }^{\mathrm{a}}$, J. Schaffner-Bielich ${ }^{\mathrm{b}}$, H. Stöcker ${ }^{\dagger}$, and W. \\ Greiner $^{\mathrm{a}}$ \\ ${ }^{\text {a} I n s t i t u t ~ f u ̈ r ~ T h e o r e t i s c h e ~ P h y s i k, ~ D-60054 ~ F r a n k f u r t ~ a m ~ M a i n, ~ G e r m a n y ~}$ \\ ${ }^{\mathrm{b}}$ Riken BNL Research Center, Brookhaven National Lab, Upton, New York 11973
}

Compelling evidence for a new form of matter has been claimed to be formed in $\mathrm{Pb}+\mathrm{Pb}$ collisions at SPS. We critically review two suggested signatures for this new state of matter: First the suppression of the $\mathrm{J} / \Psi$, which should be strongly suppressed in the QGP by two different mechanisms, the color-screening [1] and the QCD-photoeffect [2]. Secondly the measured particle, in particular strange hadronic, ratios might signal the freeze-out from a quark-gluon phase.

\section{1. $\mathrm{J} / \Psi$ suppression}

The QCD factorization theorem is used to evaluate the PQCD cross sections of heavy quarkonium interactions with ordinary hadrons. However, the charmonium states (here denoted $X$ ) are not sufficiently small to ignore nonperturbative QCD physics. Thus, we evaluate the nonperturbative QCD contribution to the cross sections of charmoniumnucleon interaction by using an interpolation between known cross sections [3]. The $J / \Psi-N$ cross section evaluated in this paper is in reasonable agreement with SLAC data [1.

Indeed, the $A$-dependence of the $J / \Psi$ production studied at SLAC at $E_{\text {inc }} \sim 20 \mathrm{GeV}$ exhibits a significant absorption effect [4] leading to $\sigma_{a b s}(J / \Psi-N)=3.5 \pm 0.8 \mathrm{mb}$. It was demonstrated [5] that, in the kinematic region at SLAC, the color coherence effects are still small on the internucleon scale for the formation of $J / \Psi$ 's. So, in contrast to the findings at higher energies, at intermediate energies this process measures the genuine $J / \Psi-N$ interaction cross section at energies of $\sim 15-20 \mathrm{GeV}$ [5].

To evaluate the nonperturbative QCD contribution we use an interpolation formula [3] for the dependence of the cross section on the transverse size $b$ of a quark-gluon configuration Three reference points are used to fix our parametrization of the cross sections (cf. Tab. 1). The $X-N$ cross sections is calculated via: $\sigma=\int \sigma(b) \cdot|\Psi(x, y, z)|^{2} \mathrm{~d} x \mathrm{~d} y \mathrm{~d} z$, where $\Psi(x, y, z)$ is the charmonium wave function. In our calculations we use the wave functions from a non-relativistic charmonium model (see [6]).

We follow the analysis of [7] to evaluate the fraction of $J / \Psi$ 's (in $p p$ collisions) that come from the decays of the $\chi$ and $\Psi^{\prime}$. So, the suppression factor $S$ of $J / \Psi$ 's produced in the nuclear medium is calculated as:

$S=0.6 \cdot\left(0.92 \cdot S^{J / \Psi}+0.08 \cdot S^{\Psi^{\prime}}\right)+0.4 \cdot S^{\chi}$. Here $S^{X}$ are the respective suppression factors

\footnotetext{
$\dagger$ Invited Speaker

${ }^{*}$ This work was supported by Deutsche Forschungsgemeinschaft (DFG), Bundesministerium für Bildung und Forschung (BMBF), Gesellschaft für Schwerionenforschung (GSI) and Graduiertenkolleg Theoretische und Experimentelle Schwerionenphysik. One of us (LG) is supported by the Josef Buchmann Foundation.
} 
Table 1

\begin{tabular}{|c|c|c|c|c|}
\hline$c \bar{c}$-state & $\mathrm{J} / \Psi$ & $\Psi^{\prime}$ & $\chi_{c 10}$ & $\chi_{c 11}$ \\
\hline$\sigma(\mathrm{mb})$ & 3.62 & 20.0 & 6.82 & 15.9 \\
\hline
\end{tabular}

The total quarkonium-nucleon cross sections $\sigma$. For the $\chi$ two values arise, due to the spin dependent wave functions $(l m=10,11)$.

of the different pure charmonium states $X$ in nuclear matter. The $S^{X}$ are for minimum bias $p A$ collisions within the semiclassical approximation (cf. [8]).

The charmonium states are produced as small configurations, then they evolve to their full size. Therefore, if the formation length of the charmonium states, $l_{f}$, becomes larger than the average internucleon distance, one has to take into account the evolution of the cross sections with the distance from the production point [5].

The formation length of the $J / \Psi$ is given by $l_{f} \approx \frac{2 p}{m_{\Psi^{\prime}}^{2}-m_{J / \Psi}^{2}}$, where $p$ is the momentum of the $J / \Psi$ in the rest frame of the target. For a $J / \Psi$ produced at midrapidity at SPS energies, this yields $l_{f} \approx 3 \mathrm{fm}$. Due to the lack of better knowledge, we use the same $l_{f} \approx 3 \mathrm{fm}$ for the $\chi$. For the $\Psi^{\prime}$ we use $l_{f} \approx 6 \mathrm{fm}$, because it is not a small object, but has the size of a normal hadron, i.e. the pion. For $E_{l a b}=800 \mathrm{AGeV}$ we get a factor of two for the formation lengths due to the larger Lorentz factor.

However, this has a large impact on the $\Psi^{\prime}$ to $J / \Psi$-ratio depicted in Fig. 1, which shows the ratio $0.019 \cdot S_{\Psi^{\prime}} / S_{J / \Psi}$ calculated with (squares $(200 \mathrm{GeV})$ and triangles $(800 \mathrm{GeV})$ ) and without (crosses) expansion. The factor 0.019 is the measured value in $p p$ collisions, because the experiments do not measure the calculated value $S_{\Psi^{\prime}} / S_{J / \Psi}$ but $\frac{B_{\mu \mu} \sigma\left(\Psi^{\prime}\right)}{B_{\mu \mu} \sigma(J / \Psi)}$. $B_{\mu \mu}$ are the branching ratios for $J / \Psi, \Psi^{\prime} \rightarrow \mu \mu$.

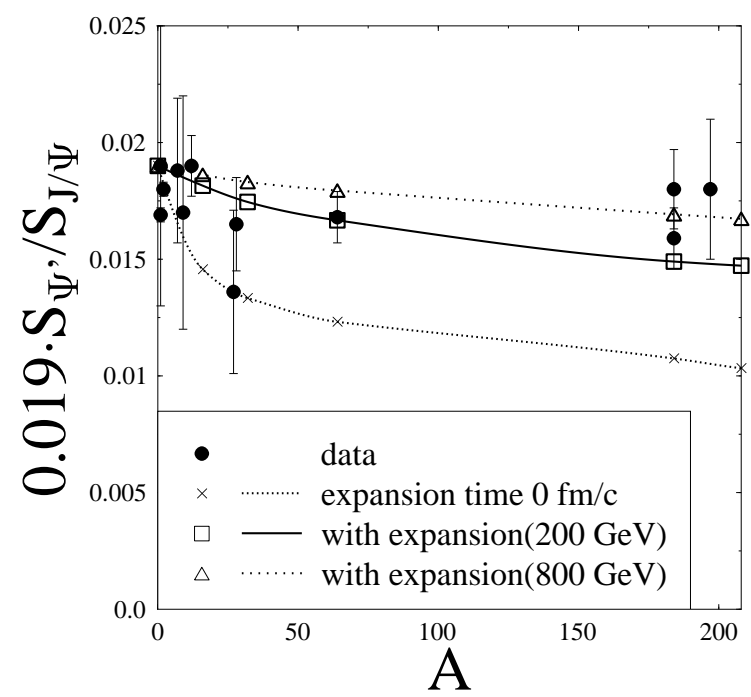

Figure 1.

The ratio $0.019 \cdot S_{\Psi^{\prime}} / S_{J / \Psi}$ is shown in $p A$ (crosses) in comparison to the data (circles). The squares and the triangles shows the ratio calculated with the expansion of small wave packages (see text).

The calculations which take into account the expansion of small wave packages show better agreement with the data (circles) (taken from [9]) than the calculation without expansion time, i.e. with immediate $J / \Psi$ formation, $l_{f}=0$. We calculated this effect both at $E_{l a b}=200 \mathrm{AGeV}$ and $800 \mathrm{AGeV}$. The data have been measured at different energies $\left(E_{l a b}=200,300,400,450,800 \mathrm{GeV}\right.$ and $\left.\sqrt{s}=63 \mathrm{GeV}\right)$. One can see that this ratio is nearly constant in the kinematical region of the data, but it decreases at smaller 
momentum (e.g. $E_{l a b}=200 \mathrm{AGeV}$ and $y<0$ ) due to the larger cross section of the $\Psi^{\prime}$.

However, the P-states yield two vastly different cross sections (see Tab. 1) for $\chi_{10}$ and $\chi_{11}$, respectively. This leads to a higher absorption rate of the $\chi_{11}$ as compared to the $\chi_{10}$. This new form of color filtering is predicted also for the corresponding states of other hadrons; e.g. for the bottomium states which are proposed as contrast signals to the $J / \Psi$ 's at RHIC and LHC!

Furthermore it is important to also take into account comoving mesons. Therefore we use the UrQMD model [10,11]. Particles produced by string fragmentation are not allowed to interact with other hadrons - in particular with a charmonium state - within their formation time (on average, $\tau_{F} \approx 1 \mathrm{fm} / \mathrm{c}$ ). However, leading hadrons are allowed to interact with a reduced cross section even within their formation time. The reduction factor is $1 / 2$ for mesons which contain a leading constituent quark from an incident nucleon and $2 / 3$ for baryons which contain a leading diquark.

Figure 2 shows the $J / \psi$ to Drell-Yan ratio as a function of $E_{T}$ for $\mathrm{Pb}+\mathrm{Pb}$ interactions at $160 \mathrm{GeV}$ compared to the NA50 data [12,13]. The normalization of $B_{\mu \mu} \sigma(J / \psi) / \sigma(\mathrm{DY})=$ 46 in $p p$ interactions at $200 \mathrm{GeV}$ has been fit to $\mathrm{S}+\mathrm{U}$ data within a geometrical model [7]. The application of this value to our analysis is not arbitrary: the model of Ref. [7] renders the identical $E_{T}$-integrated $J / \psi$ survival probability, $S=0.49$, as the UrQMD calculation for this system. An additional factor of 1.25 14 has been applied to the $\mathrm{Pb}+\mathrm{Pb}$ calculation in order to account for the lower energy, $160 \mathrm{GeV}$, since the $\mathrm{J} / \psi$ and Drell-Yan cross sections have different energy and isospin dependencies.

The gross features of the $E_{T}$ dependence of the $J / \psi$ to Drell-Yan ratio are reasonably well described by the model calculation. No discontinuities in the shape of the ratio as a function of $E_{T}$ are predicted by the simulation. The new high $E_{T}$ data [13] decreases stronger than the calculation. This could be caused by underestimated fluctuations of the multiplicity of secondaries in the UrQMD model. This occurs, since high $E_{T}$-values are a trigger for very central events with a secondary multiplicity larger than in average [15].

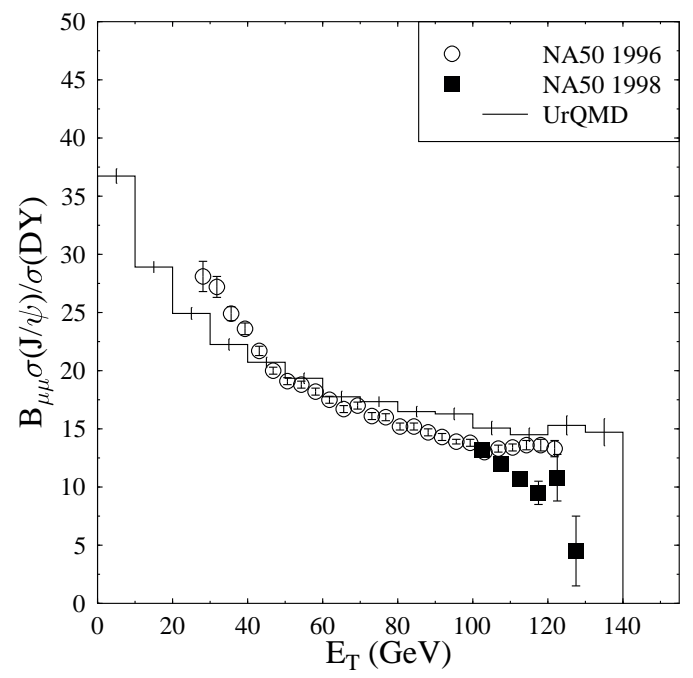

Figure 2.

The ratio of $J / \psi$ to Drell-Yan production as a function of $E_{T}$ for $\mathrm{Pb}+\mathrm{Pb}$ at $160 \mathrm{GeV}$ (see text). 


\section{Particle production}

Ideal gas model calculations have been used for a long time to calculate particle production in relativistic heavy ion collisions, e.g. [16 21]. Fitting the particle ratios as obtained from those ideal gas calculations to the experimental measured ratios at SIS, AGS and SPS for different energies and different colliding systems yields a curve of chemical freezeout in the $T-\mu$ plane. Now the question arises, how much the deduced temperature and chemical potentials depend on the model employed. Especially the influence of changing hadron masses and effective potentials should be investigated, as has been done for example in [22 25]. This is of special importance for the quest of a signal of the formation of a deconfined phase, i.e. the quark-gluon plasma. As deduced from lattice data [26], the critical temperature for the onset of a deconfined phase coincides with that of a chirally restored phase. Chiral effective models of QCD therefore can be utilized to give important insights on signals from a quark-gluon plasma formed in heavy-ion collisions.

Therefore we compare experimental measurements for $\mathrm{Pb}+\mathrm{Pb}$ collisions at SPS with the ideal gas calculations and results obtained from a chiral $\mathrm{SU}(3)$ model [27,25]. This effective hadronic model predicts a chiral phase transition at $T \approx 150 \mathrm{MeV}$. Furthermore the model predicts changing hadronic masses and effective chemical potentials, due to strong scalar and vector fields in hot and dense hadronic matter, which are constrained by chiral symmetry from the QCD Lagrangean.

In 18 the ideal gas model was fitted to particle ratios measured in $\mathrm{Pb}+\mathrm{Pb}$ collisions at SPS. The lowest $\chi^{2}$ is obtained for $T=168 \mathrm{MeV}$ and $\mu_{q}=88.67 \mathrm{MeV}$. Using these values as input for the chiral model leads to dramatic changes. As can be seen in Figure 3 . There are two main reasons for the strong deviations. First, since the chosen temperature

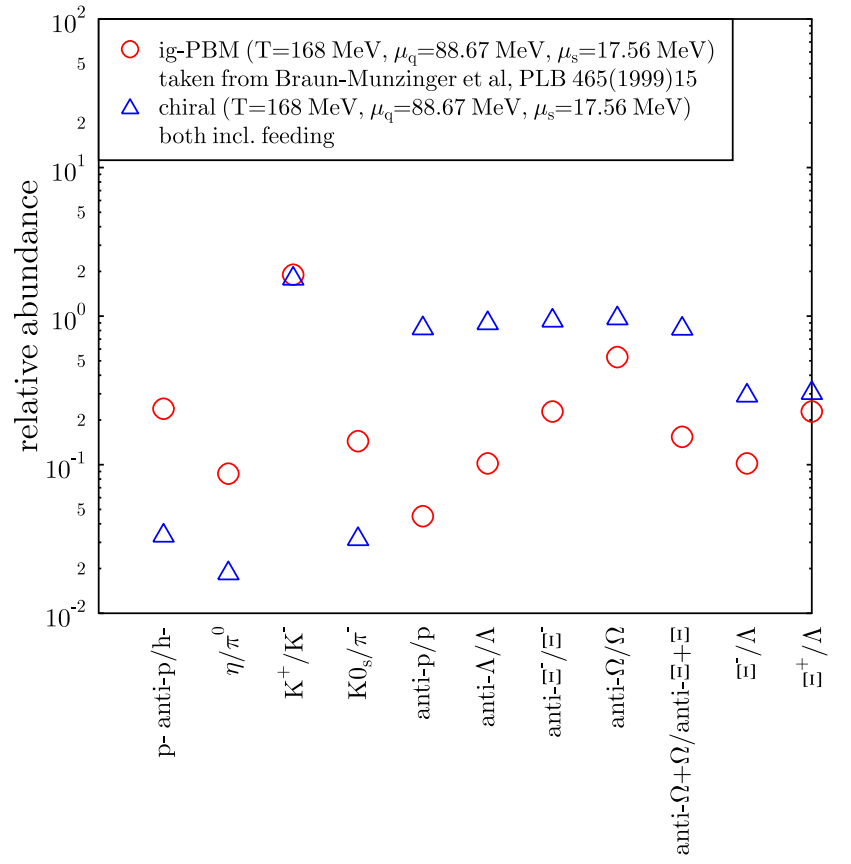

Figure 3.

Comparison of a chiral calculation with 18 for $T=$ $168 \mathrm{MeV}, \mu_{\mathrm{q}}=88.67 \mathrm{MeV}$. The huge differences result from the dropping baryonic masses, depending on the strangeness content of the particles, and from the change of the effective potentials in the chirally restored phase.

lies above the chiral phase transition temperature of the model, the effective masses of the baryons are lowered dramatically (see Figure 1). The second reason are the strongly 


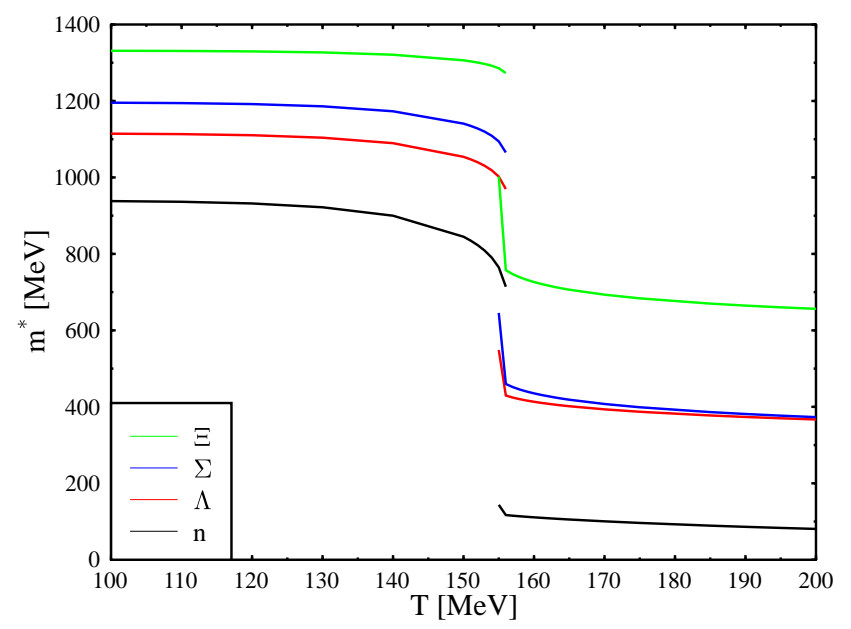

Figure 4.

Baryon masses as function of temperature for vanishing chemical potential. One sees that a phase-transition occurs at $T_{c} \approx$ $150 \mathrm{MeV}$. There the masses drop significantly and then nearly saturate. The baryon mass above $T_{c}$ scales with the strangeness content of the corresponding baryon.

changed effective potentials. While the chemical potential of the proton with the chosen parameters is $\mu_{p}=266 \mathrm{MeV}$ in the ideal gas model, the effective chemical potenial of the proton in the chiral model is only $\mu_{p} \approx 8 \mathrm{MeV}$, due to the reduction by the vectorfield. Such a small effective chemical potential leads to nearly equal particle-antiparticle numbers and therefore to tremendeously enhanced particle-antiparticle ratios.

Obviously, the freeze-out temperature and chemical potential have to be readjusted to account for the in-medium effects of the hadrons in the chiral model.

We call the best fit the parameter set that gives a minimum in the value of $\chi^{2}$, with

$$
\chi^{2}=\sum_{i} \frac{\left(r_{i}^{\text {exp }}-r_{i}^{\text {model }}\right)^{2}}{\sigma_{i}^{2}} .
$$

Here $r_{i}^{e x p}$ is the experimental ratio, $r_{i}^{\text {model }}$ is the ratio calculated in the model and $\sigma_{i}$ represents the error in the experimental data points as quoted in [18]. We included the following ratios in the fit procedure: $\frac{\bar{p}-p}{h^{-}}, \frac{\eta}{\pi^{0}}, \frac{K^{+}}{K^{-}}, \frac{K_{s}^{0}}{\pi^{-}}, \frac{\bar{p}}{p}, \frac{\bar{\Lambda}}{\Lambda}, \frac{\bar{\Xi}}{\Xi}, \frac{\bar{\Omega}}{\Omega}, \frac{\bar{\Omega}+\Omega}{\Xi^{-}+\Xi^{-}}, \frac{\Xi^{-}}{\Lambda}, \frac{\Xi^{+}}{\Lambda}$. The resulting values of $\chi^{2}$ for different $T-\mu$ pairs are shown in figure 5 . In all calculations $\mu_{s}$ was chosen such that the overall net strangeness $f_{s}$ is zero. The best values for the parameters are $T=144 \mathrm{MeV}$ and $\mu_{q} \approx 95 \mathrm{MeV}$. While the value of the chemical potential does not change much compared to the ideal gas calculation, the value of the temperature is lowered by more than $20 \mathrm{MeV}$. Furthermore Figure 5 shows, that the dropping effective masses and the reduction of the effective chemical potential make the reproduction of experimentally measured particle ratios as seen at CERN's SPS within this model impossible for $T>T_{c}$. Only for temperatures below $T_{c}$ we obtain reasonable fits. Using the best fit parameters we compare the particle ratios, as calculated in the chiral model, with the ideal gas calculation of [18] and data as compiled in [18. This is shown in figure 6. One obtains a satisfactory agreement over several magnitudes. The $\chi^{2}$ values of the chiral model is $\chi_{\text {chiral }}^{2}=26.5$. This is larger than the value in the ideal gas model of [18] $\left(\chi_{i g}^{2}=13\right)$. Note that in [18] weak decays are accounted for. If we use our ideal gas calculation without feeding from weak decays we obtain only slightly changed best values $\left(T=168, \mu_{q}=82 M e V\right)$ compared to 18 and $\chi_{i g-F F M}^{2}=21.6$. This shows that the chiral and ideal gas analysis using the same feeding procedures yield 


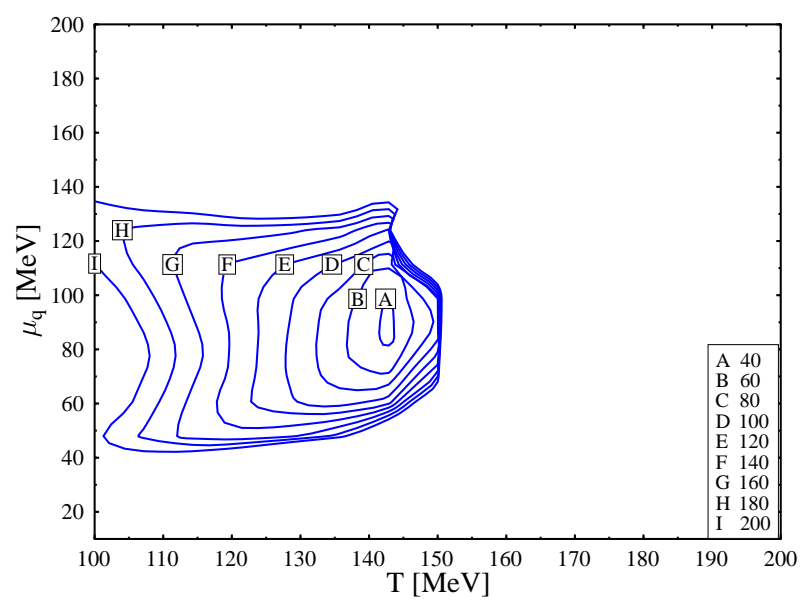

Figure 5 .

$\chi^{2}$ for chiral model, data taken from [18]. The best fit parameters are $T=144 \mathrm{MeV}$ and $\mu_{q} \approx$ $95 \mathrm{MeV} . \mu_{q}$ is chosen such that $f_{s}=0$. For $T>T_{c}$ no agreement with data from CERN SPS lead on lead collisions can be obtained.

comparable agreement with data concerning the value of $\chi^{2}$. Figure 6 shows that there is satisfactorily agreement between data and experiment. We want to emphasize, that in

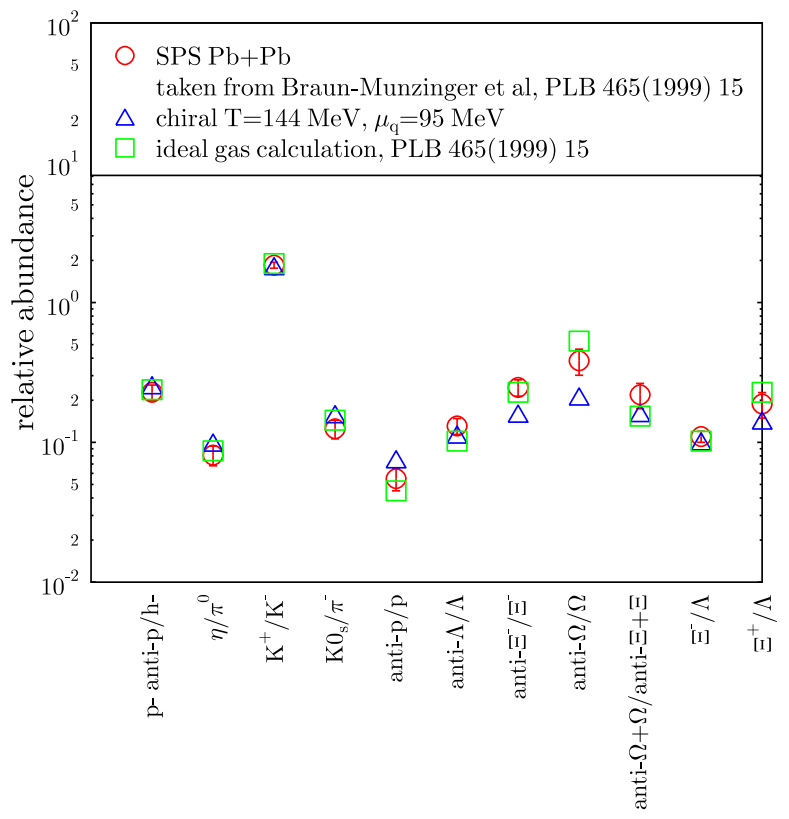

Figure 6 .

Comparison of chiral model for $T=144 \mathrm{MeV}$ compared to data and ideal gas results from 18 .

spite of the strong assumption of thermal and chemical equilibrium the obtained values for $T$ and $\mu$ differ significantly depending on the underlying model, i.e. whether and how effective masses and effective chemical potentials are accounted for. In our model, we conclude that the observed particle ratios as measured at CERNs SPS do not signal the freeze-out from a chirally restored quark-gluon phase. Note that we assume implicitly, that the particle ratios are determined by the medium effects and freeze out during the late stage expansion - no flavor changing collisions occur anymore, but the hadrons can take the necessary energy to get onto their mass shall by drawing energy from the fields. Rescattering effects will alter our conclusion but are presumably small when the chemical potentials are frozen. 


\section{REFERENCES}

1. T. Matsui and H. Satz, Phys. Lett. B178, 416 (1986).

2. D. Kharzeev, Nucl. Phys. A610, 418c (1996).

3. L. Gerland, L. Frankfurt, M. Strikman, H. Stocker, and W. Greiner, Phys. Rev. Lett. 81, 762 (1998).

4. R. L. Anderson et al., Phys. Rev. Lett. 38, 263 (1977).

5. G. R. Farrar, L. L. Frankfurt, M. I. Strikman, and H. Liu, Phys. Rev. Lett. 64, 2996 (1990).

6. L. Frankfurt, W. Koepf, and M. Strikman, Phys. Rev. D54, 3194 (1996).

7. D. Kharzeev, C. Lourenco, M. Nardi, and H. Satz, Z. Phys. C74, 307 (1997).

8. C. Gerschel and J. Hufner, Phys. Lett. B207, 253 (1988).

9. C. Lourenco, Nucl. Phys. A610, 552c (1996).

10. S. A. Bass et al., Prog. Part. Nucl. Phys. 41, 225 (1998).

11. C. Spieles, R. Vogt, L. Gerland, S. A. Bass, M. Bleicher, H. Stocker and W. Greiner, Phys. Rev. C60 (1999) 054901 [hep-ph/9902337].

12. A.Romana et al., in Proceedings of the XXXIIIrd Rencontres de Moriond, March 1998, Les Arcs, France.

13. M. C. Abreu et al., Phys. Lett. B477, 28 (2000).

14. R. Vogt, Phys. Rept. 310, 197 (1999).

15. A. Capella, E. G. Ferreiro, and A. B. Kaidalov, hep-ph/0002300 (2000).

16. D. Hahn and H. Stöcker, Nucl. Phys. A452, 723 (1986).

17. D. Hahn and H. Stöcker, Nucl. Phys. A476, 718 (1988).

18. P. Braun-Munzinger, J. Heppe, and J. Stachel, Phys. Lett. B 465, 15 (1999).

19. J. Rafelski and J. Letessier, nucl-th/9903018 (1999).

20. F. Becattini, J. Cleymans, A. Keranen, E. Suhonen, and K. Redlich, hep-ph/0002267 (2000).

21. G. D. Yen and M. I. Gorenstein, Phys. Rev. C59, 2788 (1999).

22. H. Stöcker and W. Greiner, Z. Phys. A 286, 121 (1978).

23. J. Theis, H. Stöcker, and J. Polonyi, Phys. Rev. D 28, 2286 (1983).

24. J. Schaffner, I. N. Mishustin, L. M. Satarov, H. Stöcker, and W. Greiner, Z. Phys. A341, 47 (1991).

25. D. Zschiesche, P. Papazoglou, S. Schramm, C. Beckmann, J. Schaffner-Bielich, H. Stöcker, and W. Greiner, Springer Tracts in Modern Physics 163, 129 (2000).

26. F. Karsch, hep-lat/9903031 (1998).

27. P. Papazoglou, D. Zschiesche, S. Schramm, J. Schaffner-Bielich, H. Stöcker, and W. Greiner, Phys. Rev. C 59, 411 (1999). 